\section{Patient*innen mit ent- zündlicher Arthritis und SARS-CoV-2-Infektion}

Haberman RH et al. COVID-19 in Patients With Inflammatory Arthritis: A Prospective Study on the Effects of Comorbidities and Disease-Modifying Antirheumatic Drugs on Clinical Outcomes. Arthritis Rheumatol 2020; 72: 1981-1989

Ärzt* innen der WARCOV-Studiengruppe charakterisierten Krankenhausaufenthalts- und Sterblichkeitsraten bei Patient*innen mit entzündlicher Arthritis (IA), die an einer SARS-CoV-2-Infektion erkrankten. Zusätzlich analysierten sie Zusammenhänge von Komorbiditäten und immunmodulatorischer Medikamenteneinnahme mit den Infektionsergebnissen.

WARCOV ist eine prospektive Kohortenstudie bei Patient ${ }^{*}$ innen mit immunvermittelte inflammatorischen Krankheiten (IMID), einschließlich entzündlicher Gelenkerkrankung (IA), Psoriasis und entzündlicher Darmerkrankung (IBD). Für ihre Analyse werteten die Autor ${ }^{*}$ innen klinische Daten und demografische Merkmalen von Personen mit rheumatoider Arthritis und Spondyloarthritis und symptomatischer COVID-19-Infektion aus. Darüber hinaus sammelten sie Informationen zur Erhaltungstherapie, zum Krankheitsverlauf und den Ergebnissen der Therapie. Die Studie erfolgte prospektiv, mithilfe eines webbasierten Fragebogens. Danach fanden einzelne Telefonanrufe statt und die elektronische Patientenakte wurde überprüft. Die Merkmale zu Studienbeginn und der Medikamentengebrauch wurden für hospitalisierte und ambulante Patienten zusammengefasst. Verglichen wurde das Outcome mit den verschiedenen Medikamentenklassen.

Insgesamt 103 IA-Patient* innen mit bestätigter COVID-19-Krankheit $(\mathrm{n}=80)$ und mit Verdacht auf eine Erkrankung ( $n=23)$ wurden in die Studie eingeschlossen. Das Durchschnittsalter lag bei 52,7 Jahren und der Anteil weiblicher Patienten erreichte knapp $72 \%$. Insgesamt 76 Patient*innen wurden ambulant behandelt und 27 (26\%) hospitalisiert, 4 Patient*innen (4\%) verstarben. Die Studienteilnehmer* innen wurden durchschnittlich $42 \pm 9$ Tagen nachbeobachtet.
Die Patient* innen, die im Krankenhaus behandelt wurden, waren signifikant häufiger älter $(p<0,001)$. So lag der Anteil der Patient* innen im Alter von 18-44 Jahren bei $3 \%$, aber derjenigen über 65 Jahren bei $41 \%$. Bei denjenigen, die verstarben, betrug das mittlere Alter 70 (62-87) Jahre.

Die hospitalisierten Patient*innen hatten häufiger eine komorbide Hypertonie $(p=$ $0,001)$ und eine chronisch obstruktive Lungenerkrankung $(p=0,02)$ und es zeigte sich bei ihnen ein nicht signifikanter Trend zu einem höheren BMI $(p=0,08)$.

Die dauerhafte Einnahme oraler Glukokortikoide war signifikant häufiger bei hospitalisierten im Vergleich zu ambulant behandelten Patient ${ }^{*}$ innen nachweisbar $(p<0,001)$. Bei denjenigen, die dauerhaft mit biologischen Antizytokin-Therapien behandelt wurden, fand sich dieser Zusammenhang nicht.

FAZIT

Bei Patient*innen mit entzündlicher Arthritis (IA), die an einer SARS-CoV-2Infektion erkrankten, fielen die Ergebnisse bei denjenigen, die mit Glukokortikoide therapiert wurden schlechter aus, als bei denjenigen, die mit Antizytokinen dauerhaft behandelt wurden. Das Autorenteam empfiehlt weitere Untersuchungen, um herauszufinden, ob immunmodulatorische Therapien die COVID-19-Inzidenz beeinflussen.

Richard Kessing, Zeiskam 